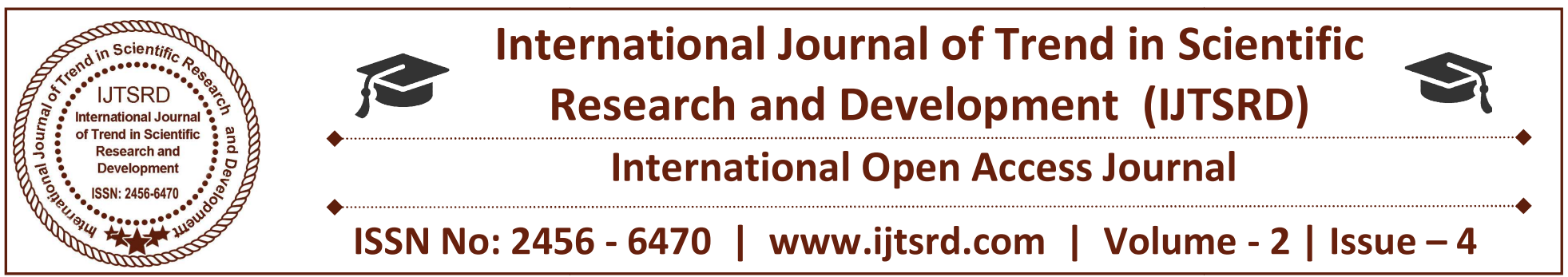

\title{
Traditional Databases vs NOSQL
}

\author{
Owais Noor Trumboo ${ }^{1}$, Jasra Nisar ${ }^{2}$ \\ M.Tech Scholars, Department of Computer Science and Engineering, \\ Punjab Technical University, India
}

\begin{abstract}
Database is a collection of logically related data and is composed of collection of files that are linked in a way that information from one of files may be combined with information from other files so that user can get exact information. Database management system (DBMS) is package that is used for creating and managing databases. A Database Management System provides a systematic way for users and programmers to create, retrieve, update and manage data. Because of DBMS it is possible for end users to create, read, update and delete the data in database. With the introduction of new technological approaches like IOT, Cloud Computing the traditional database approach is replaced by new approach known as NOSQL.
\end{abstract}

Keywords- DBMS, DBA,SQL, MYSQL, Mongo database, Cassandra

\section{INTRODUCTION}

As we know that database is the back end of the application. DBMS receives instructions from a database administrator (DBA) and instructs the system to make the changes. These commands can be load, retrieve or modify existing data from system. A database management system provides data independence. Any change in formats and storage mechanism are performed without modifying the entire application. DBMS relieves users of framing programs for data maintenance. Fourth-generation query languages, such as SQL, are used along with the DBMS package to interact with a database. Some DBMS examples are: MySQL, SQL Server, Oracle etc.
There are four main types of database architectures:

- Relational Database: In relational database, Data is organized as logically independent tables. Relationships among tables are shown through shared data. The data in one table may reference similar data in other tables, which maintains the integrity of the links among them. This feature is referred to as referential integrity. Operations such as "select" and "join" can be performed on these tables.

- Flat Database: In flat database, Data is organized in a single kind of record with a fixed number of fields. This type of database encounters more errors due to the repetitive nature of data.

- Object-Oriented Database: In this type of database, Data is organized with similarity to object-oriented programming concepts. An object consists of data and methods, while classes group objects having similar data and methods.

Hierarchical Database: In this, Data is organized with hierarchical relationships. If the one-to-many relationship is violated it becomes a complex network.

\section{RELATIONAL DATABASE AND ITS TYPES}

Relational databases use Structured Query Language and can manage a variety of transaction oriented applications. In relational database, data is present in tabular form. Today, many enterprise DBMS'S employ relational databases, but they can handle 
minimal amount of data to scale. Relational databases are the most common database systems. These databases include SQL Server, Oracle Database, Sybase, Informix, and MySQL. Relational Database Management Systems feature much better performance for managing data over desktop database programs. For example, they allow multiple users to work with the data at the same time, creating advanced security for access the data. RDBMS systems store data in columns and rows, which in turn make up tables. A set of tables makes up a schema.

The most popular relational databases are:

- MYSQL: MYSQL is an open source relational database management system based on Structured Query Language (SQL). It runs on all platforms, including Linux, Unix, and Windows.

- Oracle: Oracle database is a multi- model database management system produced and marketed by Oracle Corporation.

- Microsoft SQL Server: It is an RDBMS that supports a wide variety of transaction processing.

- DB2: It is also an RDBMS designed to store, analyse, and retrieve data efficiently.

III. NON-RELATIONAL DATABASE AND ITS TYPES

Non-relational databases are also called NOSQL databases. Some of the most known NOSQL or nonrelational Databases are MongoDB, Document DB, Cassandra, Couch base, Neo4j.The innovative structures for storing data today are NOSQL and object-oriented databases. These do not follow the table/row/column approach of RDBMS. Instead, they build bookshelves of elements and allow access per bookshelf. So, instead of tracking individual words in books, NOSQL and object-oriented databases narrow down the data you are looking for by pointing you to the bookshelf. NOSQL specifically attempts to simplify bookshelves by storing data in a demoralized way this means storing it in large chunks.A major advantage of the non-relational database is that it can easily incorporate and store all kinds of Big Data, including unstructured and semistructured data. In non-relational database, design is generally simpler and allows users to perform operations faster. Non-relational databases can scale automatically. They are becoming increasingly popular for use with real-time web applications.
In general, NOSQL databases are much more helpful than relational databases storing a wide variety of data types and sources, including social and sensor data, mobile device. A modern database has ability to allow organisations to do useful things with their data.

The most popular non- relational databases are:

- Mongo DB: It stores data in flexible, JSON- like documents, means that fields can vary from document to document and structure of data can be changed over time.

- Document DB: It is also known as documentoriented database is designed to store semistructured data as documents.

- Cassandra: It is highly scalable, high performance distributed database that is designed to handle large amounts of data across many servers. It is a type of NOSQL database.

IV. DIFFERENCE BETWEEN RELATIONAL AND NON-RELATIONAL DATABASES

Relational databases like MySQL, PostgreSQL and SQLite3 represent and store data in tables and rows. These databases are based on a branch of algebraic set theory that is known as relational algebra. Meanwhile, non-relational databases like MongoDB represent data in collections of JSON documents. The Mongo import utility can import these file formatsi.e. JSON, CSV and TSV. Mongo query targets of data are represented as BSON (binary JASON).

Mongo is a popular non-relational database for MongoDB Ember Angular and Node.js (MEAN) stack developers because it is basically written in JavaScript. JSON is JavaScript Object Notation, which is a lightweight data interchange format. If your data model turns out to be very complex, nonrelational databases like Mongo may be the best way to go. Other reasons for choosing a non-relational database include:

- Need to store serialized arrays in JSON objects.

- Storing records are in the same collection that has different fields or attributes.

- Finding yourself de-normalizing your database schema or coding around performance and horizontal scalability issues. 
- Problems are easily pre-defining your schema because of the nature of your data model.

\section{CONCLUSION}

Databases play a very important role in application development and have different models available. We are using Relational Databases from decades for different applications due to features like easy to use, simple, reliable and open source but now experts are facing number of challenges due to deployment of Traditional Relational Databases like Big Data, IOT and Cloud. The paper introduces new era of databases which are Non-Relational and minimizes the issues facing during implementation of Relational Databases.

\section{REFERENCES}

1. NaseerGaniee, "NOSQL: The Big Data Solution", International Journal of Advancement in Engineering Technology, Management and Applied Sciences, Volume 1, Issue 2, July 2014.
2. NaseerGaniee, "New Database Constraints and Modern Applications", IJLTEMAS, Volume III, Issue II, February 2014.

3. AmeyaNayak, Anil Poriya, and DikshayPoojary, "Types of NOSQL Databases and its comparison with the Relational Databases", International Journal of Applied Information Systems, Volume 5 No. 4, March 2013, www.ijais.org.

4. Paolo Atzeni, "The Relational is dead, SQL is dead, and I don't feel so good myself', Volume 42, No 2, June 2013.

5. A B Moniruzzaman and Syed AkhtarHossain, "NOSQL Database: New Era of Databases for Big Data Analytics- Classification, Comparison and Characteristics", International Journal of Database Theory and application.

6. "Database - Definition of database by MerriamWebster". Webster.com.

7. "A Relational Database Overview". oracle.com.

8. "NoSQL (Not Only SQL)". "NoSQL database, also called Not Only SQL"

9. "Jepsen: MongoDB stale reads". Aphyr.com. 2015-04-20. Retrieved 2017-03-06 